\title{
The Soft Material Obtained from an Europium (III)-Containing Ionic Liquid
}

\author{
Huifang Shao, Yige Wang, and Dan Li \\ School of Chemical Engineering and Technology, Hebei University of Technology, Tianjin 300130, China \\ *E-mail: wangyige@hrbut.edu.cn \\ Received December 23, 2010, Accepted January 19, 2011
}

\begin{abstract}
The addition of organic ligand ( $\beta$-diketone or heterocyclic compound) to the europium (III)-containing ionic liquid resulted in bright luminescent soft materials with the molar ratio of europium/ionic liquid (IL)/ligand being 1:3:1 that exhibit bright red light under UV lamp. The luminescent properties such as emission features and lifetime of ${ }^{5} \mathrm{D}_{0} \mathrm{Eu}^{3+}$ excited level are dependent on the organic ligands. The materials were characterized by FT-IR and luminescence spectroscopy. The data shows that at least parts of the ILs (carboxylic acid) are replaced with $\beta$-diketone ligand rather than the formation of europium complex with the molar ratio of $\mathrm{Eu}^{3+}$ : IL: ligand being 1:3:1, while no ILs could be replaced by the heterocyclic ligand such as Bpy and Phen.
\end{abstract}

Key Words : Ionic liquid, Europium (III), Luminescence, Organic ligand

\section{Introduction}

Ionic liquid have been an active area of research due to the inherent properties, such as negligible vapor pressures, wide liquid ranges, good thermal stabilities, considerable electric conductivities and wide electrochemical windows, which have been shown to be advantageous for a large number of applications. ${ }^{1,2}$

Metal-containing ionic liquids are regarded as promising new materials that combine the properties of ionic liquid with magnetic, photophysical/optical, or catalytic properties depending on the enclosed metal used. ${ }^{3}$ Lanthanide-containing ionic liquids are highly interesting as advanced catalysts because of their excellent Lewis acidity ${ }^{4}$ and as promising soft luminescent materials for use in photochemistry and spectroscopy due to their narrow emission bands and long radiative lifetimes of the excited state..$^{5-15}$ However, the solubility of lanthanide compounds in ionic liquids is generally rather low. Ionic liquids containing high concentrations of lanthanides can find potential applications in electrodeposition, catalysis, or photophysics. ${ }^{15}$ It is thus attractive and desirable to develop ionic liquids containing high concentrations of lanthanides. Only limited papers about the higher concentration of the lanthanide ions in ionic liquid have been reported. ${ }^{12,15,17-19}$ Incorporating lanthanide ions as an anion into ionic liquids is an effective way to increase the concentration of lanthanides. ${ }^{13,14,16}$ An alternative approach to increase the solubility of metal salts is to use task-specific ionic liquids (TSIL) bearing a complexing moiety. ${ }^{20}$

Recently, we have reported that carboxyl group-functionalized ionic liquid can react with lanthanide oxides to form a carboxylate lanthanide complex, which is transparent at room temperature. In this case, the molar ratio of ionic liquid (IL) to $\mathrm{Eu}^{3+}$ ions is to 3:1. Further addition of one equivalent molar of organic ligand is desirable in consideration of the high coordination number of lanthanide ions. Furthermore, the organic ligand can sensitize the luminescence of $\mathrm{Eu}^{3+}$ ions to result in bright red emission. The question raised here is if the organic ligand is coordinated to the carboxylate lanthanide complexes or replaces the ionic liquid from the coordinated sphere of lanthanide ions. In this work, we are trying to answer this question by using two types of organic ligands ( $\beta$-diketone and heterocyclic compounds). The organic ligands and ionic liquid used in this study are shown in Figure 1.

\section{Experimental Section}

All solvents and reagents were used as received without further purification. Europium oxide (99\%, ALDRICH), 3Bromopropanoic acid (98\%, ALDRICH), 1-Butylimidazole (98\%, ALDRICH), Ethanol (99\%, ALDRICH), 4,4',4"-trifluoro-1-(2-naphthyl)-1,3-butanedione (NTA) $(98+\%$, Matrix Scientific), 2,2-bipyridyl (Bpy) (99\%, kRs), 1,10phenanthroline (Phen) (99\%, CHEMICAL REAGENT), J\&KCHEMICALLTD). 3-(5-Carboxypropyl)-1-butylimidazolium bromide (IL) was prepared according the previously reported procedure. ${ }^{21}$

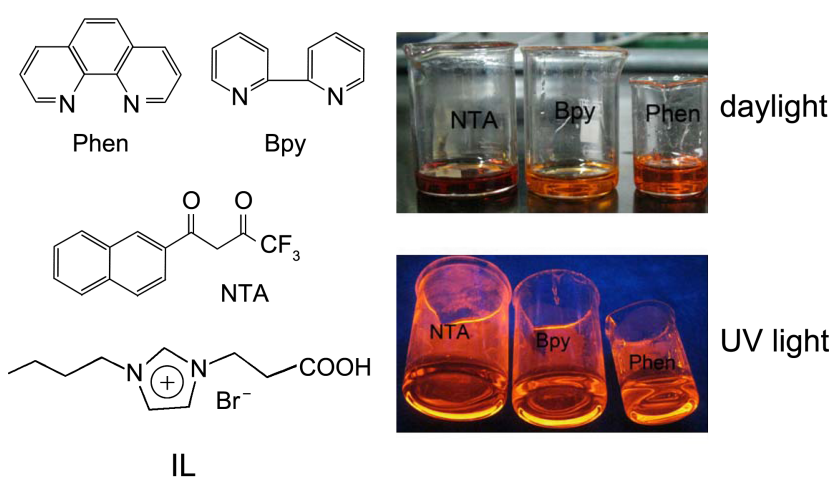

Figure 1. The Ionic liquid (IL), organic ligand and the photographs of europium-containing ionic liquids. 

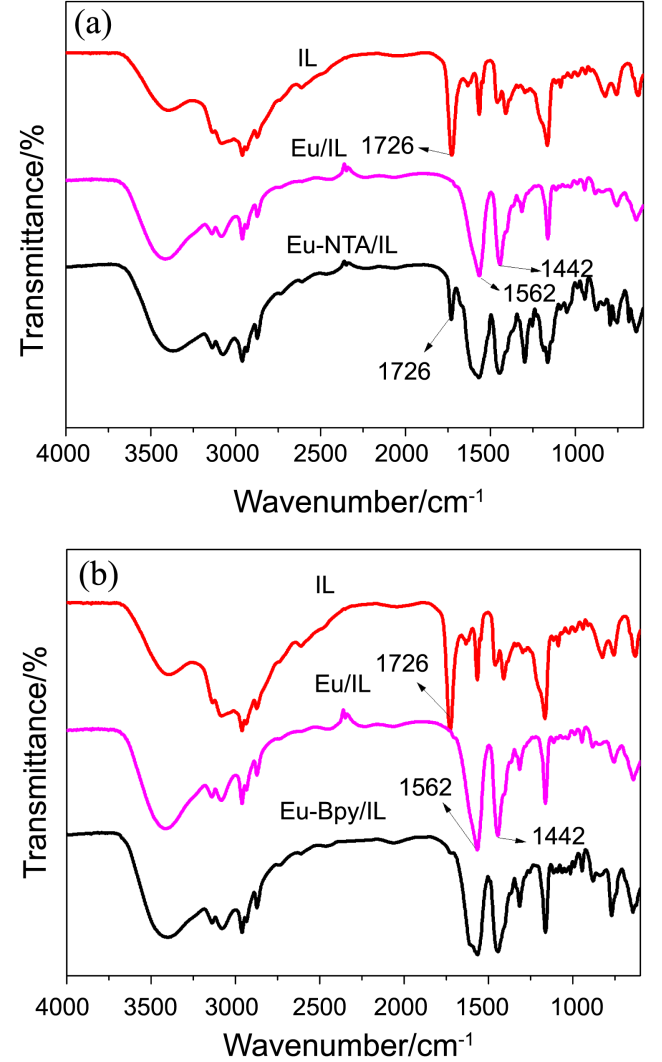

Figure 2. FT-IR spectra of IL, Eu/IL, Eu-NTA/IL and IL-EuBpy. The molar ratio of Eu/IL/NTA (Bpy) is 1:3:1.

Infrared (IR) spectra were obtained on a Bruker Vector 22 spectrometer using $\mathrm{KBr}$ pellets for solid samples, from $400-4000 \mathrm{~cm}^{-1}$ at a resolution of $4 \mathrm{~cm}^{-1}$ (16 scans collected). The steady-state luminescence spectra and the lifetime measurements were measured on an Edinburgh Instruments FS920P spectrometer, with a $450 \mathrm{~W}$ xenon lamp as the steady-state excitation source, a double excitation monochromator (1800 lines $\mathrm{mm}^{-1}$ ), an emission monochromator (600 lines $\mathrm{mm}^{-1}$ ) and a Hamamatsu RMP928 photomultiplier tube. All spectra were obtained at room temperature.

Preparation of Eu/IL. To the ionic liquid (IL) $(0.6 \mathrm{mmol})$ containing ca. $5 \mathrm{~mL}$ of water, $\mathrm{Eu}_{2} \mathrm{O}_{3}(0.11 \mathrm{mmol})$ was added. The stirred mixture was heated at $100{ }^{\circ} \mathrm{C}$ for $12 \mathrm{~h}$. The excess of $\mathrm{Eu}_{2} \mathrm{O}_{3}$ was removed by filtration. After removal of water by rotary evaporation, a transparent and slightly yellowish ionic liquid (Eu/IL) with significantly increased viscosity was obtained and further dried in vacuum at 60 overnight.

Preparation of Eu-NTA/IL (Eu-Phen/IL, Eu-Bpy/IL).

General Procedure: To a solution of Eu/IL $(0.2 \mathrm{mmol})$ in Ethanol $(20 \mathrm{~mL})$, organic ligands (NTA or Bpy or Phen) $(0.2$ mmol) was added. The stirred mixture was refluxed overnight and the ethanol was then evaporated to obtain highly viscously transparent europium complex-containing ionic liquids (Eu-NTA/IL, Eu-Bpy/IL and Eu-Phen/IL (Fig. 1)). The molar ratio of $\mathrm{Eu}^{3+} / \mathrm{IL} /$ organic ligand was 1:3:1.
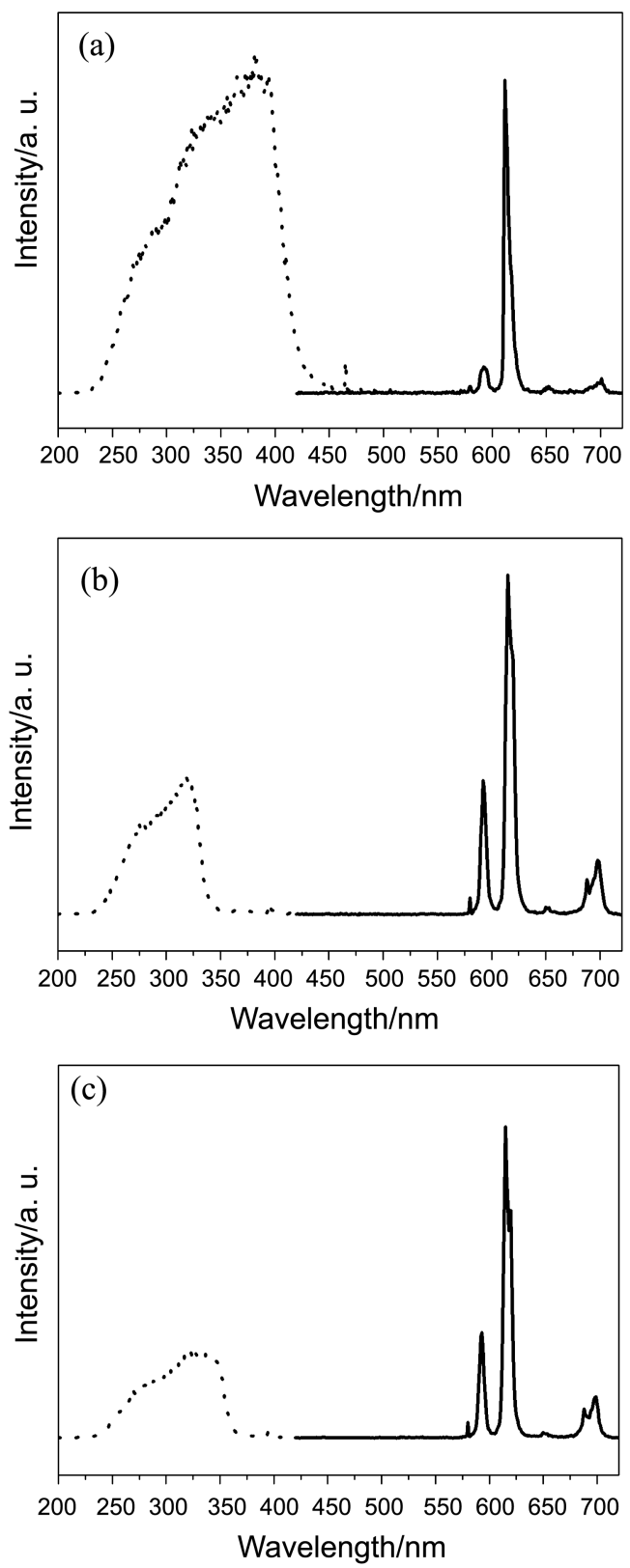

Figure 3. Excitation (dot line) and emission spectra (solid line) of Eu-NTA/IL (a), Eu-Bpy/IL (b) and Eu-Phen/IL (c).

\section{Results and Discussion}

The formation of europium carboxylate complex $(\mathbf{E u} / \mathbf{I L})$ between IL and $\mathrm{Eu}^{3+}$ ions can be easily obtained by reacting 3-(5-carboxypropyl)-1-butylimidazolium bromide with $\mathrm{Eu}_{2} \mathrm{O}_{3}$ in the presence of an appropriate amount of $\mathrm{H}_{2} \mathrm{O}$ as reported previously. ${ }^{15}$ Evaporating $\mathrm{H}_{2} \mathrm{O}$ leads to highly viscous complex which is transparent at room temperature. Refluxing the mixture of Eu/IL and organic ligands (NTA, Bpy,Phen) in ethanol solution overnight leads to highly viscous transparent europium complex-containing ionic liquids, Eu-NTA/IL, Eu-Bpy/IL and Eu-Phen/IL (Fig. 1). The molar ratio of $\mathrm{Eu}^{3+} / \mathrm{IL} /$ organic ligand was 1:3:1. 
Figure 2 shows the FT-IR spectra of the samples. From Figure 2 we can find no absorption band at $1726 \mathrm{~cm}^{-1}$ from - $\mathrm{COOH}$ group can be observed for $\mathbf{E u} / \mathbf{I L}$, indicating the ionic liquids are coordinated with $\mathrm{Eu}^{3+}$ ions, which can also be revealed by the presence of bands at 1562 and $1442 \mathrm{~cm}^{-1}$ attributing to the asymmetric and the symmetric stretch of the carboxylate group, respectively. Interestingly, the absorption band at $1726 \mathrm{~cm}^{-1}$ reappears upon the addition of the organic ligand NTA (Fig. 2a), indicating that at least parts of the ILs (carboxylic acid) are replaced with NTA ligand rather than the formation of europium complex with the molar ratio of $\mathrm{Eu}^{3+}$ :IL:NTA being 1:3:1, the same result can be obtained with another diketone ligand 2-thenoyltrifluoroacetone (here not shown). Heterocyclic ligands such as Phen and Bpy were also involved in this study because they can also coordinate to $\mathrm{Eu}^{3+}$ ions and sensitize the luminescence of them. No band at $1726 \mathrm{~cm}^{-1}$ corresponding to the $-\mathrm{COOH}$ group from the ILs can be observed when heterocyclic ligand was used as shown in Figure $2 b$, this means that they cannot replace the IL in the complexes.

The excitation and emission spectra of Eu-NTA/IL are shown in Figure 3a. The excitation spectrum obtained by monitoring the ${ }^{5} \mathrm{D}_{0} \rightarrow{ }^{7} \mathrm{~F}_{2}$ transition at $612 \mathrm{~nm}$ displays a broad excitation band ranging from 200 to $420 \mathrm{~nm}$, which may result from the $\pi \rightarrow \pi^{*}$ transitions of the organic ligands. A weak sharp $\mathrm{f}-\mathrm{f}$ transition line at $465 \mathrm{~nm}\left({ }^{5} \mathrm{~F}_{0} \rightarrow\right.$ ${ }^{5} \mathrm{D}_{2}$ ) can be also observed. The relatively low intensity of the intra- $4 \mathrm{f}^{6}$ transitions compared with that of the broad band indicates an energy transfer from the ligands to the central $\mathrm{Eu}^{3+}$ ions. Excitation of the ligands $(370 \mathrm{~nm})$ leads to only sharp emission peaks arising from transitions between ${ }^{5} \mathrm{D}_{0}$ $\rightarrow{ }^{7} \mathrm{~F}_{\mathrm{J}}$ crystal-field components $(J=0,1,2,3,4)$ with the hypersensitive transition ${ }^{5} \mathrm{D}_{0} \rightarrow{ }^{7} \mathrm{~F}_{2}$ line as the most prominent line, suggesting that the $\mathrm{Eu}^{3+}$ ion site indeed lack a center of inversion. ${ }^{22}$ The typical red color of europium emission is mostly attributed to the strongest transition ${ }^{5} \mathrm{D}_{0}$ $\rightarrow{ }^{7} \mathrm{~F}_{2}$ centered at $612 \mathrm{~nm}$. The presence of the forbidden ${ }^{5} \mathrm{D}_{0} \rightarrow{ }^{7} \mathrm{~F}_{1}$ transition around $592 \mathrm{~nm}$ implies that $\mathrm{Eu}^{3+}$ ions are in a coordination sphere with low symmetry. ${ }^{23}$ The luminescence decay curve is found to be mono-exponential and the luminescence decay time of ${ }^{5} \mathrm{D}_{0}$ level is $0.27 \mathrm{~ms}$.

The excitation and emission spectra of Eu-Bpy/IL and Eu-Phen/IL are shown in Figure $3 b$ and 3c, respectively. The excitation spectra display a broad excitation band resulting from the $\pi \rightarrow \pi^{*}$ transitions of the heterocyclic ligands superimposed with sharp line of the intra- $4 \mathrm{f}^{6}$ transitions, this indicates that an energy transfer occurs from the ligands to the central $\mathrm{Eu}^{3+}$ ions. Excitation at the ligands $(300 \mathrm{~nm})$ leads to sharp emission peaks arising from transitions between ${ }^{5} \mathrm{D}_{0} \rightarrow{ }^{7} \mathrm{~F}_{\mathrm{J}}$ crystal field components $(J=0,1$, $2,3,4)$. The typical red color of europium emission is mostly attributed to the strongest transition ${ }^{5} \mathrm{D}_{0} \rightarrow{ }^{7} \mathrm{~F}_{2}$ centered at $614 \mathrm{~nm}$. Furthermore, the detection of a single line for the ${ }^{5} \mathrm{D}_{0} \rightarrow{ }^{7} \mathrm{~F}_{0}$ transition, the local-field splitting of the ${ }^{7} \mathrm{~F}_{1,2}$ levels and the higher intensity of the ${ }^{5} \mathrm{D}_{0} \rightarrow{ }^{7} \mathrm{~F}_{2}$ transition indicate that the $\mathrm{Eu}^{3+}$ local coordination site has a low symmetry without an inversion center. The ${ }^{5} \mathrm{D}_{0}$ lifetime value of Eu-Bpy/IL and Eu-Phen/IL is determined to be $0.71 \mathrm{~ms}$ and $0.83 \mathrm{~ms}$, respectively, from the decay curve that can be well-reproduced by means of a single-exponential function. It is worth to note that the lifetime of ${ }^{5} \mathrm{D}_{0} \rightarrow \mathrm{Eu}^{3+}$ excited level of Eu-Bpy/IL and Eu-Phen/IL is much longer than that of the corresponding pure complex $\left(\mathrm{Eu}(\mathrm{bpy})_{2} \cdot 2 \mathrm{H}_{2} \mathrm{O}\right.$, $\left.\mathrm{Eu}(\mathrm{Phen})_{2} \cdot 2 \mathrm{H}_{2} \mathrm{O}\right)$ and sol-gel-derived materials. ${ }^{24,25}$ One reasonable explanation for this might be that both bipyridine (or phenanthroline) molecules and carboxylate groups from IL are coordinated $\mathrm{Eu}^{3+}$ ions, thus, most of the water molecules deactivating the excited state of $\mathrm{Eu}^{3+}$ ions in the first coordination sphere can be expelled. ${ }^{26}$

In summary, luminescent soft materials were obtained by addition of organic ligands (NTA, Bpy or Phen) into a carboxyl-functionalized ionic liquid where $\mathrm{Eu}^{3+}$ ions are coordinated to the oxygen atoms of carboxylate groups. All the luminescent soft materials show bright red emission under UV-light irradiation due to the energy transfer from the organic ligands to $\mathrm{Eu}^{3+}$ ions. FT-IR and luminescence spectra indicate that at least parts of the ILs (carboxylic acid) are replaced with NTA ligand rather than the formation of europium complex with the molar ratio of $\mathrm{Eu}^{3+}: \mathrm{IL}: \mathrm{NTA}$ being 1:3:1 whereas no IL could be replaced by the heterocyclic ligand such as Bpy and Phen. The new soft materials display attractive photophysical properties in the visible region. Therefore, the materials are not only of great interest from an academic viewpoint, but they might also be extremely valuable materials for various applications.

Acknowledgments. This work is financially supported by the National Natural Science Foundation of China (No. 20871040, No. 20901022), Hebei Province Natural Science Foundation for Distinguished young Scholar (No. B2010000034), Program for New Century Excellent Talents in University (NCET-09-0113), Tianjin Natural Science Foundation (09JCYBJC05700), the Key Project of Chinese Minstry of Education (208016), and Hebei Province Natural Science Foundation (2009000013).

\section{References}

1. Plechkova, N. V.; Seddon, K. R. Chem. Soc. Rev. 2008, 37, 123.

2. Anastas, P. T.; Warner, J. C. Oxford University Press: New York, 1998.

3. Mallick, B.; Balke, B. Angew. Chem. Int. Ed. 2008, 47, 1.

4. Ross, J.; Xiao, J. L. Green Chem. 2002, 4, 129.

5. Mudring, A. V.; Babai, A.; Arenz, S.; Giernoth, R.; Binnemans, K.; Driesen, K.; Nockemann, P. J. Alloy Compd. 2006, 418, 204.

6. Nockemann, P.; Beurer, E.; Driesen, K.; Deun, R. V.; Hecke, K. V.; Meervelt, L. V.; Binnemans, K. Chem. Commun. 2005, 4354.

7. Guillet, E.; Imbert, D.; Scopelliti, R.; Bunzli, J.-C. G. Chem. Mater. 2004, 16, 4063.

8. Lunstroot, K.; Driesen, K.; Nockemann, P.; Goerller-Walrand, C.; Vioux, A. Chem.Mater. 2006, 18, 5711.

9. Babai, A.; Mudring, A.-V. Chem. Mater. 2005, 17, 6230.

10. Gaillard, C.; Billard, I.; Chaumont, A.; Mekki, S.; Denecke, M. A.; Moutiers, G. Inorg. Chem. 2005, 44, 8355.

11. Samikkanu, S.; Mellem, K.; Berry, M.; May, P. S. Inorg. Chem. 2007, 46, 7121.

12. Li, H. R.; Shao, H. F.; Wang, Y. G.; Qin, D. S.; Liu, B. Y.; Zhang, 
W. J. Chem. Commun. 2008, 5209.

13. Tang, S.; Babai, A.; Mudring, A.-V. Angew. Chem. Int. Ed. 2008, 47, 7631

14. Mallick, B.; Balke, B.; Felser, C.; Mudring, A.-V. Angew. Chem. Int. Ed. 2008, 47, 7635 .

15. Li, H. R.; Liu, P.; Shao, H. F.; Wang, Y. G.; Zheng, Y. X.; Chen, Y. H. J. Mater. Chem. 2009, 19, 5533.

16. Nockemann, P.; Thijs, B.; Postelmans, N.; Van Hecke, K. J. Am. Chem. Soc. 2006, 128, 13658.

17. Nockemann, P.; Thijs, B.; Pittois, S.; Thoen, J.; Glorieux, C.; Kirchner, B. J. Phys. Chem. B 2006, 110, 20978.

18. Nockemann, P.; Thijs, B.; Parac-Vogt, T. N.; Van Hecke, K.; Nguyen, M. T. Inorg. Chem. 2008, 47, 9987.

19. Nockemann, P.; Thijs, B.; Lunstroot, K.; Parac-Vogt, T. N.; Deun,
R. V. Chem. Eur. J. 2009, 1449.

20. Olivier, J. H.; Camerel, F.; Selb, J.; Retailleau, P.; Ziessel, R. Chem. Commun. 2009, 1133.

21. Dubreuil, J. F.; Bazureau, J. P. Tetrahedron Lett. 2000, 41, 7351.

22. Lenaerts, P.; Driesen, K.; Deun, R. V.; Binnemans, K. Chem. Mater. 2005, 17, 2148.

23. Julian, B.; Corberan, R.; Cordoncillo, E.; Escribano, P.; Viana, B. J. Mater. Chem. 2004, 14, 3337.

24. Li, H. R.; Lin, N. N.; Wang, Y. G.; Feng, Y.; Gan, Q. Y.; Zhang, H. J. Eur. J. Inorg. Chem. 2009, 519.

25. Li, H. R.; Lin, J.; Zhang, H. J.; Fu, L. S.; Meng, Q. G.; Wang, S. B. Chem. Mater. 2002, 14, 3651 .

26. Feng, Y.; Li, H. L.; Gan, Q. Y.; Wang, Y. G.; Liu, B. Y.; Zhang, H. J. J. Mater. Chem. 2010, 20, 972. 